\title{
A mathematical model of mechanotransduction reveals how mechanical memory regulates mesenchymal stem cell fate decisions
}

\author{
Tao Peng ${ }^{1 \dagger}$, Linan Liư ${ }^{2 \dagger}$, Adam L MacLean ${ }^{1}$, Chi Wut Wong ${ }^{2}$, Weian Zhao ${ }^{2}$ and Qing Nie ${ }^{1 *}$ (1)
}

\begin{abstract}
Background: Mechanical and biophysical properties of the cellular microenvironment regulate cell fate decisions. Mesenchymal stem cell (MSC) fate is influenced by past mechanical dosing (memory), but the mechanisms underlying this process have not yet been well defined. We have yet to understand how memory affects specific cell fate decisions, such as the differentiation of MSCs into neurons, adipocytes, myocytes, and osteoblasts.

Results: We study a minimal gene regulatory network permissive of multi-lineage MSC differentiation into four cell fates. We present a continuous model that is able to describe the cell fate transitions that occur during differentiation, and analyze its dynamics with tools from multistability, bifurcation, and cell fate landscape analysis, and via stochastic simulation. Whereas experimentally, memory has only been observed during osteogenic differentiation, this model predicts that memory regions can exist for each of the four MSC-derived cell lineages. We can predict the substrate stiffness ranges over which memory drives differentiation; these are directly testable in an experimental setting. Furthermore, we quantitatively predict how substrate stiffness and culture duration co-regulate the fate of a stem cell, and we find that the feedbacks from the differentiating MSC onto its substrate are critical to preserve mechanical memory. Strikingly, we show that re-seeding MSCs onto a sufficiently soft substrate increases the number of cell fates accessible.

Conclusions: Control of MSC differentiation is crucial for the success of much-lauded regenerative therapies based on MSCs. We have predicted new memory regions that will directly impact this control, and have quantified the size of the memory region for osteoblasts, as well as the co-regulatory effects on cell fates of substrate stiffness and culture duration. Taken together, these results can be used to develop novel strategies to better control the fates of MSCs in vitro and following transplantation.
\end{abstract}

Keywords: Mesenchymal stem cell, ECM, YAP/TAZ, Cell fate decision, Stiffness sensing, Memory, Bistability, Nonlinear dynamics, Mathematical modeling

\section{Background}

Changes in cellular state can be regulated by mechanical signals from the cellular microenvironment, such as the local extracellular matrix (ECM) stiffness [1-4]. Recent studies into mechanotransduction have demonstrated

\footnotetext{
*Correspondence: qnie@uci.edu

${ }^{\dagger}$ Equal contributors

${ }^{1}$ Department of Mathematics, Center for Complex Biological Systems, and Center for Mathematical and Computational Biology, University of California, Irvine, CA 92697, USA

Full list of author information is available at the end of the article
}

that cells sense and integrate mechanical cues from the ECM, causing transcriptional changes to occur and influencing cell fate decisions $[1-3,5]$. Mesenchymal stem cells (MSCs) are controlled by signals from the ECM and exhibit a wide range of differential gene expression patterns $[1,6]$. The mechanisms governing how MSCs sense the surrounding ECM, and the myriad other factors affecting MSC fate, including interactions with proteins and ligands, tethering, and porosity, remain incompletely defined [3, 7]. Further understanding of 
how differentiation cues are mediated by mechanical stimuli will help to facilitate new biomaterial design, cell-based therapeutics, and engineered tissue constructs for use in regenerative medicine.

The signals arising at the stem cell/substrate interface are complex and dynamic [7], however it has been shown that stiffness alone is enough to direct MSC differentiation $[3,4]$. MSCs undergo neurogenic or adipogenic differentiation on soft substrates $(<1 \mathrm{kPa})$, and myogenic or osteogenic differentiation on stiff substrates $(>10 \mathrm{kPa})[1,5]$ (Fig. 1). Upon further study, more complex differentiation patterns emerge. For example, it has been observed that cells cultured for a period of time on stiff substrates, such as standard tissue culture polystyrene (TCPS) plates, differentiate into osteogenic lineage cells even after being transferred from the stiff to a softer substrate [8]. Seeding MSCs on a phototunable substrate demonstrates that osteogenic patterns of gene expression persist even after decreasing the stiffness of the substrate [8]. This "mechanical memory": the ability of MSCs to remember previous physical stimuli depends on both culture time and substrate stiffness (depicted in Fig. 1).

Due to mechanical memory, MSC differentiation in vitro can yield unpredictable (and undesirable) results. Mechanical memory also makes it very difficult to perform certain in vitro assays reliably, for example on extremely soft or stiff substrates, or assays with very long or short incubation periods. Such extreme culture conditions are nonetheless important to assess in order to fully elucidate the relationship between MSC fate and substrate stiffness [9]. In addition to the impracticality of performing short (i.e. seconds) or long (i.e. months) incubation experiments, experimental knock-downs of key genes involved in mechanotransduction, such as Yesassociated protein $(Y A P)$, can be lethal or highly toxic in vitro and in vivo $[10,11]$. There is thus a need for in

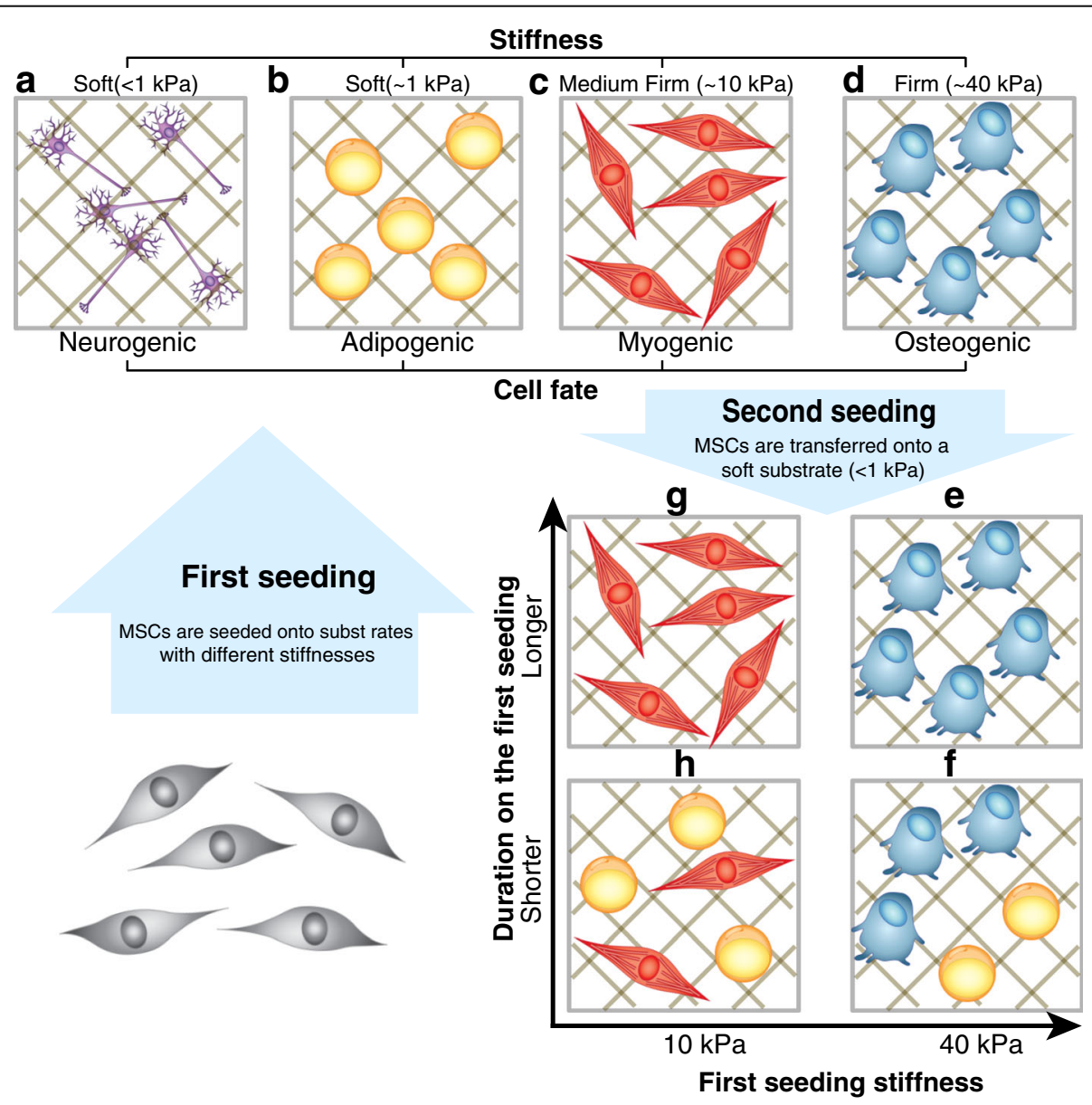

Fig. 1 Mesenchymal stem cells (MSCs) exhibit mechanical memory. $\mathbf{a}, \mathbf{b}, \mathbf{c}, \mathbf{d}$ : MSCs differentiate into distinct lineages under different substrate stiffness conditions by upregulating lineage marker genes TUBB3 ( $<1 \mathrm{kPa}$ stiffness, the neurogenic fate), PPARG ( $\sim$ kPa stiffness, the adipogenic fate), MYOD1 ( 10 kPa stiffness, the myogenic fate), or RUNX2 ( 40 kPa stiffness, the osteogenic fate). When re-seeded onto a soft substrate $(\sim 1$ $\mathrm{kPa})$, MSCs are expected to undergo adipogenic differentiation $[1,6,64]$. e, f: However, for higher first seeding stiffness values (>10 kPa), or for long first seeding durations (>10 days), mechanical memory leads to heterogeneous osteogenic differentiation [8]. $\mathbf{g}$, $\mathbf{h}$ : The model predicts that for high first seeding stiffness values $(\sim 10 \mathrm{kPa})$, or for long first seeding durations, mechanical memory leads to heterogeneous myogenic differentiation 
silico studies to simulate culture conditions and to map the MSC fate predictions to experimental results describing mechanically induced cell differentiation.

Several mathematical models of mechanotransduction have been built to describe cell differentiation directed by external mechanical stimuli $[12,13]$. These include, for example, analysis of the role of YAP/TAZ, the transcriptional factors $Y A P$ and transcriptional co-activator with PDZ-binding motif (TAZ), in mechanosensing [14], and models that aim to predict cell differentiation during bone healing $[12,15,16]$. Mousavi et al. developed a 3D mechanosensing computational model to illustrate that matrix stiffness can regulate MSC fates. Their simulation results of MSC differentiation in response to substrate stiffness are in agreement with published experimental observations [13]. Burke et al. built a computational model to test whether substrate stiffness and oxygen tension regulate stem cell differentiation during fracture healing [12]. Their model predicted the presence of major processes involved with fracture healing, including cartilaginous bridging, endosteal and periosteal bony bridging, and bone remodeling, using parameters related to cell proliferation, oxygen tension, and substrate stiffness. However, these models are limited in that the effects of regulatory factors were not considered [12-16]. Furthermore, these studies used different models to represent different experimental observations. Hence it is difficult to describe the overall cell state space and to study the transitions between cell fates [12-16]. Thus, there is a need for a dynamic mathematical model, which can stimulate a continuous range of stiffness values and their associated cell fates.

Here we present a mathematical model of MSC differentiation controlled by the following set of core mechanisms
(Fig. 2 and Table 1) [1, 6, 9]. The MSCs sense the stiffness of their environment directly via their adhesion to the substrate. The transcriptional factors YAP and TAZ mediate the signal via their interaction with downstream genes involved in cell differentiation. TUBB3, a gene encoding Tubulin beta- 3 chain tightly correlated with a neurogenic cell fate is expressed when MSCs receive stimuli from a soft stiffness environment $(<1 \mathrm{kPa})$ [1]. PPARG, peroxisome proliferator-activated receptor gamma, encodes an adipogenic marker and has been shown to be turned on in soft stiffness environments ( 1 kPa) [6]. MYOD1, myogenic differentiation 1, a myogenic gene turned on in medium-stiff environments $(\sim 10 \mathrm{kPa})$, encodes key factors regulating muscle differentiation [1]. RUNX2, runt-related transcription factor 2 , an osteogenic gene which is upregulated in high stiffness environments ( $40 \mathrm{kPa})$, is a key transcriptional factor involved in osteoblast differentiation [1] (Fig. 1). We use this set of four lineage-specific genes in our model to minimally describe the transcriptional changes observed during MSC differentiation into four distinct cell fates under the influence of mechanical stimuli mediated by $Y A P / T A Z$ signaling.

Based on the proposed regulatory network structure (Fig. 2), we simulate gene expression dynamics under different mechanical dosings. Each in silico experiment describes MSCs cultured in two passages: a first seeding and a second seeding. The substrate stiffness for the first seeding and the duration of the first seeding are particularly important in cell fate determination of MSCs. We also discover an important role for the second seeding stiffness through our simulation studies. Crucially, this two-seeding setup permits mechanical memory to be observed and studied. We assess when cell fates are determined not only by the current substrate stiffness but

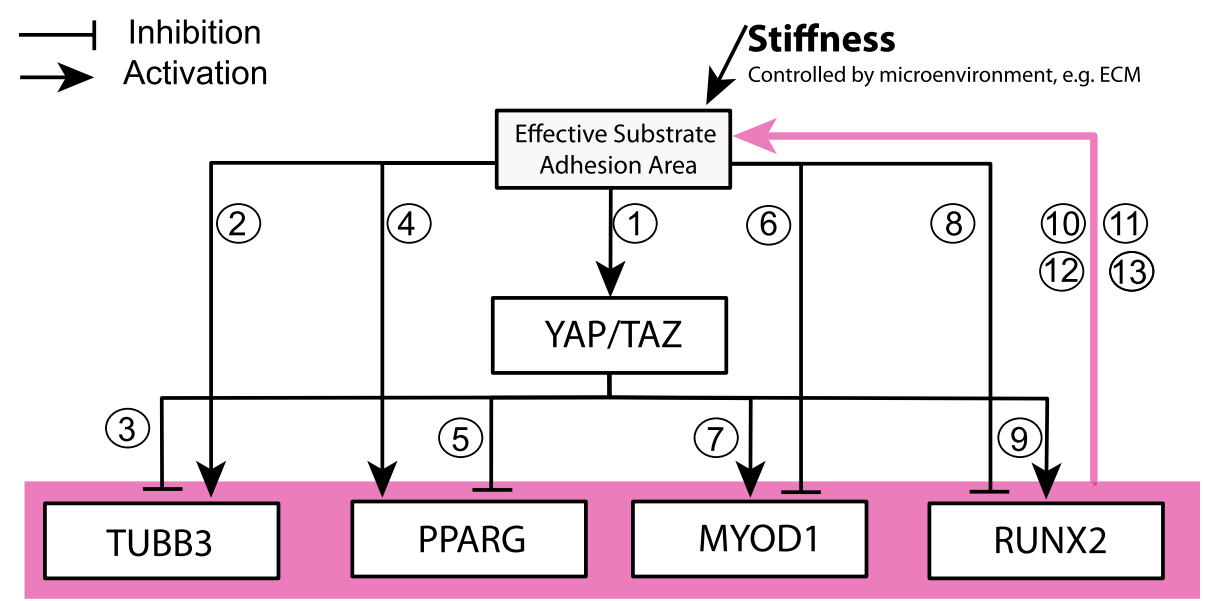

Fig. 2 Regulatory network used to construct the mathematical model. The boxes represent genes or factors involved in MSC differentiation and the lines with arrows and with bars denote gene activation and inhibition respectively. External stiffness affects the substrate adhesion area. The pink line with an arrow denotes regulations by all species within the pink box. The circled indices refer to experimental evidence for each interaction, details of which are given in Table 1 
Table 1 The references of regulatory interactions in the network

\begin{tabular}{|c|c|c|}
\hline Index of Arrows & Interactions & References \\
\hline 1 & YAP/TAZ is identified as mechanical sensors and mediators. & Halder, G et al, 2012; Dupont S. et al. 2011. [6, 18] \\
\hline 3 & The inhibition of TUBB3 can be attenuated by YAP depletion. & Alarcon, C et al. 2009 [65] \\
\hline 5 & $\begin{array}{l}\text { PPARG can be bound to } T A Z \text {, which results in } \\
\text { transcription inhibitions from the aP2 promoter. }\end{array}$ & Hong, J.H. et al, 2006.[21] \\
\hline 7 & $\begin{array}{l}\text { TAZ functions as an enhancer of MYOD-mediated myogenic } \\
\text { differentiation. }\end{array}$ & Jeong, H. et al, 2010. [66] \\
\hline 9 & RUNX2 has binding domain to TAZ for osteocalcin expression. & Hong, J.H. et al, 2006. Hong, J.H. et al, 2005 [20, 21] \\
\hline $10,11,12,13$ & $\begin{array}{l}\text { Increased cell spreading results in higher stiffness sensitivity } \\
\text { via increased binding of integrins to the ECM. }\end{array}$ & $\begin{array}{l}\text { Halder G et al, 2012. Sun Y et al, } 2012 . \\
\text { Bernabe B P et al, 2016. [6, 17, 67] }\end{array}$ \\
\hline $2,4,6,8$ & $\begin{array}{l}\text { These arrows are necessary for the dynamics of TUBB3, PPARG, } \\
\text { MYOD1, and RUNX2 on all possible stiffness environment since } \\
\text { TUBB3, PPARG, MYOD1, and RUNX2 are expressed only on the } \\
\text { super soft stiffness }(<1 \mathrm{kPa}) \text {, the soft stiffness }(\sim 1 \mathrm{kPa}) \text {, the } \\
\text { medium stiffness }(\sim 10 \mathrm{kPa}) \text {, and the high stiffness }(\sim 40 \mathrm{kPa}) \\
\text { environment respectively. }\end{array}$ & Engler, A.J. et al,2006; Halder G et al, $2012[1,6]$ \\
\hline
\end{tabular}

also by past exposure and find that a memory region exists for each of the four MSC-derived cell lineages studied. Our model demonstrates that stiffness-based MSC differentiation results from non-cooperative regulation of representative genes. Moreover, we show that lowering the second seeding stiffness of MSCs leads to a more diverse palette of MSC fates.

\section{Results}

A mathematical model based on a mechanotransduction network

The following set of biological assumptions has been used to develop the mathematical model. MSCs differentiate according to their surrounding mechanical environment $[2-4,6,17]$. Directed differentiation towards a particular lineage can be guided if the cells are cultured in a microenvironment that mimics the tissue elasticity of the environment in vivo [2, 3, 17]. Stiff substrates promote cellECM adhesion interactions via integrins [6]. These adhesive interactions control the localization of downstream transcriptional factors $Y A P$ and $T A Z$, which have been identified as mechanical sensors and mediators of such signals $[6,18]$. YAP/TAZ localizes in the cytoplasm on soft substrates $(\sim 1 \mathrm{kPa})$ and can re-localize to the nucleus on stiff substrates $(\sim 40 \mathrm{kPa})$, thus functioning as a mechanosensitive transcription factor $[6,18]$.

Additionally, YAP/TAZ has been reported to be an upstream factor of a number of genes associated with cell differentiation cues $[6,18,19]$. For example, the inhibition of TUBB3 can be attenuated by YAP depletion, whereas that the factor PPARG binding to TAZ results in inhibition of transcription from the aP2 promoter [20, 21]. $T A Z$ functions as an enhancer of $M Y O D$-mediated myogenic differentiation. RUNX2 can also bind to TAZ and cause osteocalcin to be expressed, thus promoting osteogenic differentiation $[20,21]$. To describe these interactions, we model $Y A P / T A Z$ as both a downstream factor of the mechanical stimulus from the ECM and an upstream factor of the selected cell lineage genes [1, 22] (Fig. 2 and Table 1). Previous references show an intriguing relationship between morphological changes to MSCs and their lineage differentiation potential, whereby morphological changes have been shown to be instrumental to the process of MSC differentiation [1, 17, 18, 23-25]. In particular, it was shown that MSC osteogenic differentiation is enhanced by the morphological change of MSCs and $M Y O D 1$ induced the myogenic differentiation efficiency via the morphological change of MSCs [26, 27]. Other factors regulating cell spreading such as NKX2.5 were integrated in the model implicitly [28]. Therefore, we model a feedback loop between the lineage-specific target genes and the cellular sensing of substrate stiffness.

In order to predict how mechanical dosing influences MSC differentiation, we use ordinary differential equations to model the MSC lineage regulatory network [2932] (Fig. 2 and Table 1). We assume that changes in the stiffness of the substrate act as stimulus to the cell (mediated by stiffness receptors) [12, 33]. We use Hill functions to model the chemical activation/inhibition [31, 32, 34]. We model the feedback loop that controls mechanical memory via a non-cooperative regulation, i.e., any of the lineage-specific genes (TUBB3, PPARG, MYOD1, $R U N X 2)$ can increase the effective stiffness adhesion area (we use "OR-GATE" logic). The feedback loop controls the expression of $Y A P / T A Z$ and its downstream genes via the stimulus (i.e., the change in stiffness [8]). We also test a feedback model of cooperative regulations (where TUBB3, PPARG, MYOD1 and RUNX2 must act together to increase the effective stiffness adhesion area, i.e. "AND-GATE" logic) but find that it does not satisfy 
the dynamical requirements of the MSC differentiation system (see Methods for full details).

\section{Model simulations predict mechanical memory regions for each lineage-specific gene}

The non-cooperative regulation model displays multiple steady states over the behavioral regions that we have investigated (with first seeding stiffness values ranging from $0.1 \mathrm{kPa}$ to greater than $100 \mathrm{kPa}$; Fig. 3). This range is sufficient to encompass all known in vitro studies [1, $6,8]$. In Fig. 3a and $\mathrm{b}$ the multiple steady states of $Y A P /$ $T A Z$ expression over the stiffness range studied are shown, and changes in the $Y A P / T A Z$ state can be visualized as the stiffness increases (blue lines) or decreases (red lines). The nonlinear relationship between YAP/ $T A Z$ and the stiffness of the substrate along the blue lines is consistent with previous observations $[9,19]$.
Figure 3c demonstrates bistability in the relative gene expression of TUBB3 (driver of neurogenic differentiation) downstream of $Y A P / T A Z$. TUBB3 is "OFF" when the stiffness is lower than $0.2 \mathrm{kPa}$. It will be turned "ON" as the stiffness increases to $0.25 \mathrm{kPa}$. It turns "OFF" again as the stiffness increases further. Meanwhile, TUBB3 stays "ON" when the stiffness decreases below $0.2 \mathrm{kPa}$, thus highlighting the mechanical memory observed during neurogenic differentiation. Notably, TUBB3 stays "OFF" as the stiffness decreases from $0.6 \mathrm{kPa}$. We define the region of stiffness from 0.25 to $0.55 \mathrm{kPa}$ as a "differentiation memory region" for TUBB3. This means that if the first seeding stiffness is within this range, the cell will "remember" the stiffness of this first seeding substrate, and will differentiate according (towards a neurogenic fate) upon reseeding. Our model also predicts novel differentiation memory regions for PPARG (0.6 to $3 \mathrm{kPa}$; Fig. $3 \mathrm{~d})$ and
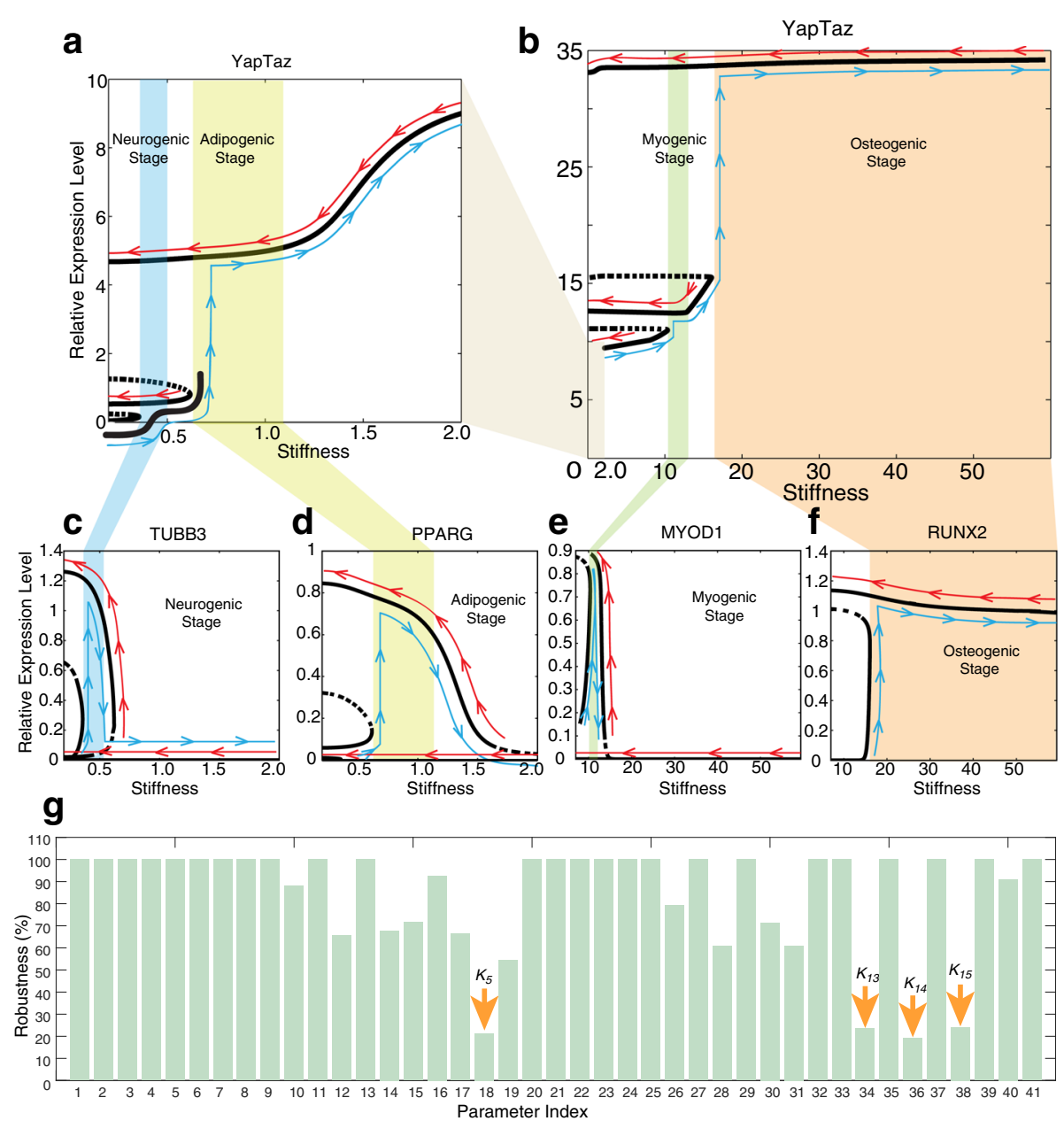

Fig. 3 Multistability in the MSC differentiation network. The relative expression level of YAP/TAZ in a stiffness range from $0.1 \mathrm{kPa}$ to $60 \mathrm{kPa}$ is shown (b), with inset (a). The relative expression levels of lineage-specific genes are shown in (c-f). On each plot the $x$-axis is the stiffness of the substrate and the $y$-axis is the relative gene expression level. Blue lines illustrate changes in the relative expression level as the stiffness increases; red lines illustrate changes in the relative expression level as the stiffness decreases. (g). The robustness of the parameters in the mathematical model. The $x$-axis is the parameter index, corresponding to the notation of Table 2. The $y$-axis is the robustness of the parameters (defined in Methods) 
MYOD1 (10 to $15 \mathrm{kPa}$; Fig. 3e). RUNX2 displays the largest differential memory region of the four lineagespecific marker genes studied.

Figure 3c-f collectively demonstrate a bistable region for each of the four lineage-specific genes studied. This is a startling prediction: that a region of mechanical memory exists for each of the cell fates, not just for osteogenic differentiation, as has been previously reported [8]. For neurogenic and adipogenic differentiation, the memory regions are smaller than that of osteoblasts yet may still be of great importance for stem cell fate regulation. The true contribution of each will require further study to elucidate, as a host of interacting factors contribute to the neurogenic and adipogenic cell fate decisions, including those which are not currently included in our model, such as the role of substrate-induced stemness and of epithelial to mesenchymal transition [35-37].

To test the robustness of the mathematical model we calculate the values of the robustness of each parameter in Eqs. (1,2,3,4,5 and 6) with respect to the memory and multistability of the system (full details of our methodology are in Methods). Out of the 41 parameters tested, 37 are robust to small changes for the majority of perturbations tested (and many of these 37 were robust more than $80 \%$ of the time) (Fig. 3g). Four parameters are found to be sensitive to small perturbations. All of these four parameters are involved in myogenic or osteogenic differentiation. Both these processes involve relatively large memory regions, thus it is possible that following these perturbations memory is maintained over parts of - but not the entire - original memory regions. Overall, we find that the system displays robustness using the parameters given in Table 2, with regard to the memory effects and the multistability of the states.

\section{A lower second seeding stiffness permits a greater number of MSC lineages}

Potential energy landscape analysis is an appealing method with which we can investigate the system and study the MSC differentiation propensities under different conditions [38-40]. Since it is not possible to write down a complete expression for the potential energy of the system, we use an approximate method derived from mean field theory in order to calculate quasi-potential in terms of the six system variables [40, 41]. Explicitly, we calculate the potential of the system as $U(X)=-\ln \left(P_{s s}(X)\right)$, where $P_{s s}(X)$ is the total probability of the state vector $X$, and $X$ describes all the states of the system [40, 41].

Table 2 Parameter values of the mathematical model

\begin{tabular}{|c|c|c|c|c|c|c|c|}
\hline Index & Parameter & Value & Estimated from references & Index & Parameter & Value & $\begin{array}{l}\text { Estimated } \\
\text { from references }\end{array}$ \\
\hline 1 & $k_{1}$ & 0.2 & {$[1,6]$} & 2 & $k_{2}$ & 2.2 & {$[1,6]$} \\
\hline 3 & $k_{3}$ & 5 & {$[1,6]$} & 4 & $k_{4}$ & 9 & {$[1,6,8]$} \\
\hline 5 & $k_{5}$ & 4 & {$[1,6]$} & 6 & $k_{6}$ & 2.9 & {$[1,6]$} \\
\hline 7 & $k_{7}$ & 3 & {$[1,6]$} & 8 & $k_{8}$ & 5 & {$[1,6,8]$} \\
\hline 9 & $k_{9}$ & 2 & {$[1,6,8]$} & 10 & $K_{1}$ & 600 & {$[1,6]$} \\
\hline 11 & $n_{1}$ & 4 & {$[1,6]$} & 12 & $K_{2}$ & 1.1 & {$[1,6]$} \\
\hline 13 & $n_{2}$ & 2 & {$[1,6]$} & 14 & $K_{3}$ & 1300 & {$[1,6]$} \\
\hline 15 & $n_{3}$ & 6 & {$[1,6]$} & 16 & $K_{4}$ & 0.8 & {$[1,6,8]$} \\
\hline 17 & $n_{4}$ & 2 & {$[1,6,8]$} & 18 & $K_{5}$ & 20,000 & {$[1,6]$} \\
\hline 19 & $n_{5}$ & 4 & {$[1,6]$} & 20 & $K_{6}$ & 1 & {$[1,6]$} \\
\hline 21 & $n_{6}$ & 20 & {$[1,6]$} & 22 & $K_{7}$ & 60,000 & {$[1,6]$} \\
\hline 23 & $n_{7}$ & 6 & {$[1,6]$} & 24 & $K_{8}$ & 1.1 & {$[1,6]$} \\
\hline 25 & $n_{8}$ & 20 & {$[1,6]$} & 26 & $K_{9}$ & 0.1 & {$[1,6]$} \\
\hline 27 & $n_{9}$ & 2 & {$[1,6]$} & 28 & $K_{10}$ & 0.5 & {$[1,6]$} \\
\hline 29 & $n_{10}$ & 8 & {$[1,6]$} & 30 & $K_{11}$ & 0.89 & {$[1,6]$} \\
\hline 31 & $n_{11}$ & 2 & {$[1,6]$} & 32 & $K_{12}$ & 4 & {$[1,6]$} \\
\hline 33 & $n_{12}$ & 8 & {$[1,6]$} & 34 & $K_{13}$ & 12 & {$[1,6]$} \\
\hline 35 & $n_{13}$ & 20 & {$[1,6]$} & 36 & $K_{14}$ & 3 & {$[1,6]$} \\
\hline 37 & $n_{14}$ & 60 & {$[1,6]$} & 38 & $K_{15}$ & 16 & {$[1,6,8]$} \\
\hline 39 & $n_{15}$ & 45 & {$[1,6,8]$} & 40 & $K_{16}$ & 4.5 & {$[1,6,8]$} \\
\hline 41 & $n_{16}$ & 55 & {$[1,6,8]$} & \multicolumn{2}{|c|}{$d_{i}(i=1,2, \cdots 6)$} & 1 & Unconstrained \\
\hline
\end{tabular}


In order to visualize this potential function we project it onto a two-dimensional plane, defined by the species in our model: $Y A P / T A Z$, and the effective stiffness adhesion area (SAA). In doing so we integrate out the four remaining system variables (TUBB3, PPARG, MYOD1, and RUNX2) [40, 41]. We are thus able to study how the potential depends on these variables for different stiffness values. In Fig. 4 we show the potential functions for four different conditions (we change the second-seeding stiffness values). Overall, we find that by reducing the second seeding stiffness, a greater number of steady states is permitted.

We simulate more than 10,000 initial conditions in order to avoid becoming trapped in local minima [40, 41]. We observe that across the entire landscape there are four stable states (or basins of attractions), representing neurogenic, adipogenic, myogenic, and osteogenic cell lineages. At a final stiffness of $\sim 0.4 \mathrm{kPa}$, MSCs can differentiate into each of the four possible lineages (Fig. 4a). Only at such sufficiently small values for the second stiffness can MSCs differentiate into neurons: the basin of attraction for the neurogenic fate (i.e. the probability of differentiating into a neuron) is the smallest of the four fates. This means that mechanical memory is observed only over a small range of space. In comparison, a much greater mechanical memory effect is seen for the osteogenic lineage, corresponding to a larger basin of attraction. Figure $4 b$ and $c$ show the potential landscapes at second seeding stiffness values of $\sim 0.8 \mathrm{kPa}$ and $\sim 12 \mathrm{kPa}$, respectively. The number of basins decreases to three, and then two, as the second seeding stiffness increases. When the second seeding stiffness increases further to $\sim 20 \mathrm{kPa}$, we have only one remaining basin of attraction, thus only one possible cell fate: in this region the largest mechanical memory effect is seen, and osteogenic differentiation dominates. These data intriguingly suggest that simply by controlling the substrate stiffness upon re-seeding we can control the number of cell fates that are accessible to MSCs.

The duration of the initial seeding determines the fate of an MSC

In addition to studying the effect of the second seeding stiffness on the fate of MSCs, we perform tests to assess the agreement between our model and in vitro observations regarding MSC differentiation [1, 18]. Specifically, we manipulate the stiffness of the second seeding substrate and the duration of the first seeding, and find, consistent with previous studies [5, 42], that both of these variables play an important role in the fate determination of an MSC upon differentiation. In addition these simulation results highlight several new phenomena.

In order to examine how the first seeding duration affects MSC fates, we use a non-dimensionalized version of the model, that is, we express time in relative units. In Fig. 5a, the first and second seeding stiffness values are $30 \mathrm{kPa}$ and $0.4 \mathrm{kPa}$, respectively. When the duration of the first seeding time is 50 (blue line), MSCs differentiate into osteoblasts (consistent with [5]): RUNX2 is the
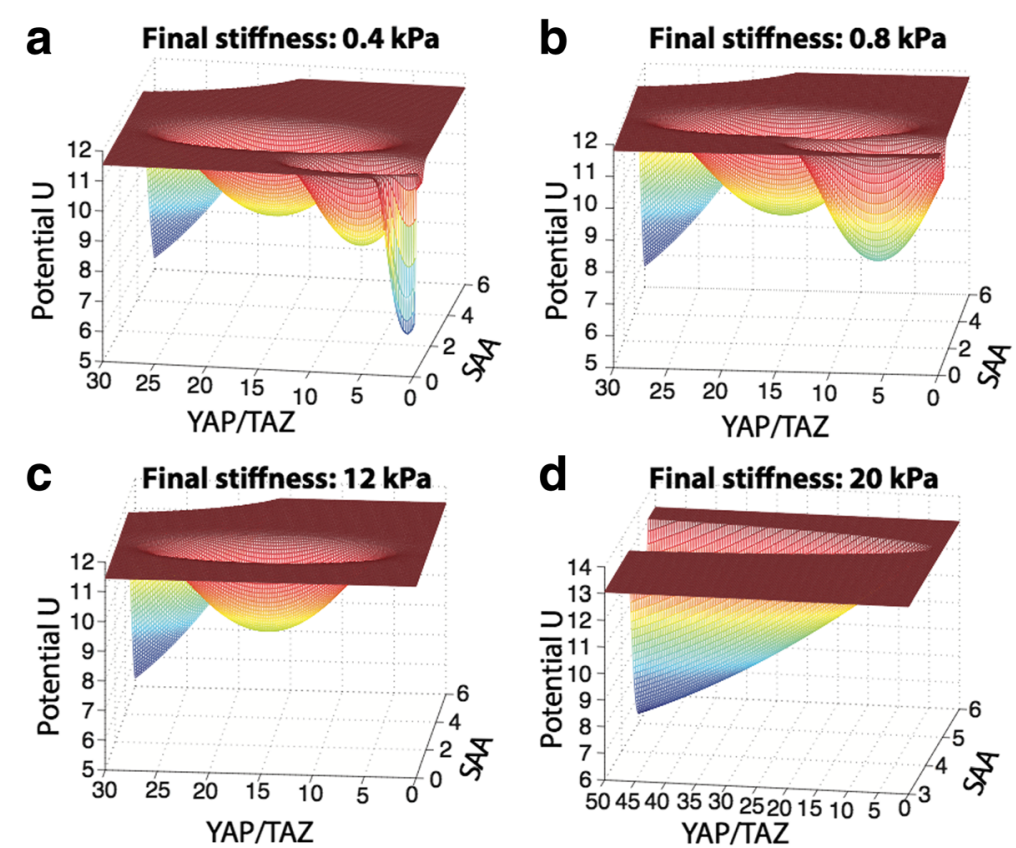

Fig. 4 Potential landscapes of the regulatory network under different stiffness conditions. In each figure the relative stiffness level (input to the system) is plotted on the $x$-axis, the relative expression level of YAP/TAZ is plotted on the $y$-axis, the energy potential function $U$ is plotted on the z-axis. Potential energy landscapes are shown with stiffness values of $\sim 0.4 \mathrm{kPa}(\mathbf{a}), \sim 0.8 \mathrm{kPa}(\mathbf{b}), \sim 12 \mathrm{kPa}(\mathbf{c})$ and $\sim 20 \mathrm{kPa}(\mathbf{d})$ 

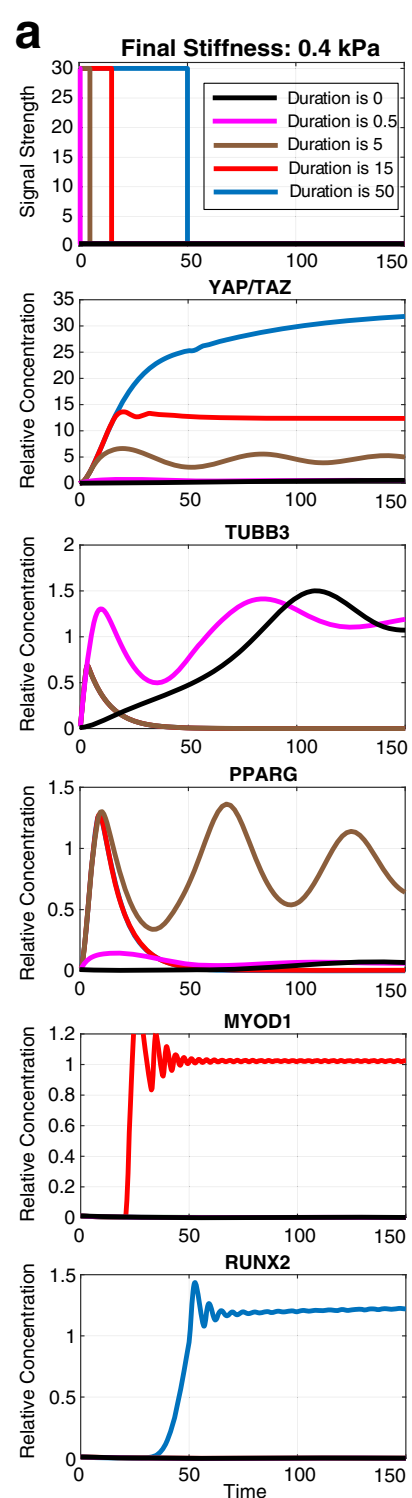
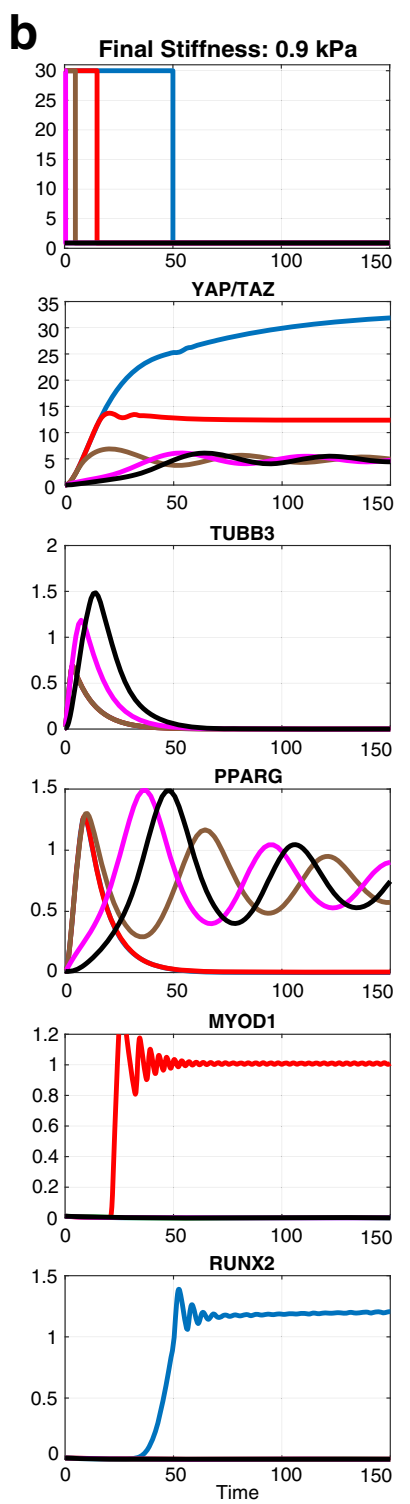
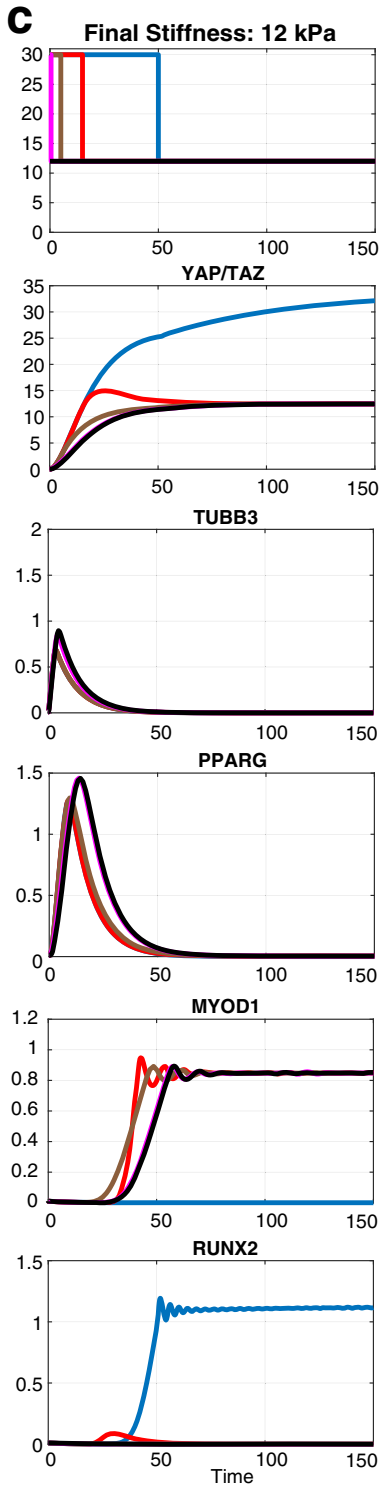

Fig. 5 The duration of the first seeding regulates MSC fates via mechanical memory. The first seeding stiffness in this figure is $30 \mathrm{kPa}$. The second seeding stiffness is $0.4 \mathrm{kPa}(\mathbf{a}), 0.9 \mathrm{kPa}(\mathbf{b})$ or $12 \mathrm{kPa}$ (c). When the duration of the first seeding is 50 (blue lines), MSCs undergo osteogenic differentiation according to memory. When the duration of the first seeding is 15 (red lines), MSCs undergo myogenic differentiation. When the duration of the first seeding is 5 (brown lines in columns A and B), MSCs differentiate into adipocytes or myogenic cells. When the duration of the first seeding is 0.5 (pink lines in column A), MSCS are able to undergo adipogenic, myogenic, or neurogenic differentiation. Finally, when the duration of the first seeding is 0 (black lines), MSCs are able to undergo adipogenic, myogenic, or neurogenic differentiation

only gene that is highly expressed under this condition. When the first seeding duration is 15 (red line), MSCs differentiate into skeletal muscle cells (MYOD1 high); when the first seeding duration is five (brown line), MSCs differentiate into adipocytes (PPARG high). Finally when the first seeding duration is 0.5 or 0 (pink and black lines), MSCs differentiate into neurogenic cells (TUBB3 high). These results are consistent with previous studies and highlight the breadth of control that mechanical memory enables: MSCs can be directed to four different fates by changing only the duration of the first seeding, keeping both of the first and the second substrate stiffness values constant. Although mechanical memory is not observed when the first seeding duration is less than 0.5 , for the first seeding durations greater than five, we predict that mechanical memory will influence MSC fates, directing MSCs towards myogenic or adipogenic lineages.

Mechanical memory persists when the second seeding stiffness increases, but the number of fates accessible to an MSC decreases, as described in previous sections. In Fig. $5 \mathrm{~b}$ the second seeding stiffness is $0.9 \mathrm{kPa}$. When the 
relative duration of the first seeding is 50 (blue line), MSCs differentiate into osteoblasts according to mechanical memory. When the relative duration of the first seeding is 15 (red line), MSCs differentiate into myocytes (again, influenced by memory). When the relative duration of the first seeding is 5, 0.5 or 0 , however (brown, pink or black lines), MSCs differentiate into adipocytes: mechanical memory is not present when the second seeding duration is less than 15.

Figure $5 \mathrm{c}$ shows the dynamics of the system when the second seeding stiffness is $12 \mathrm{kPa}$. For the longest first seeding duration (blue line), MSCs differentiate into osteoblasts, as above, but when the duration is 15 or lower (red, brown, pink or black lines), MSCs differentiate into myocytes. These data illustrate that as the second seeding stiffness increases, the range of first seeding durations over which mechanical memory is observed decreases, which is consistent with the observation from Yang et al [8]. At a second seeding stiffness of $12 \mathrm{kPa}$, the memory effect is observed only for osteogenic differentiation, and not for any other lineages. Intriguingly, higher first seeding stiffness values for shorter periods of time might accelerate an MSC towards lineage commitment. TUBB3 expression approaches the steady state quickly following stimulation on a $30 \mathrm{kPa}$ substrate for a relative time of 0.5 (Fig. $5 \mathrm{a}$, pink line). Compare this to the differentiation characteristics of an MSC seeded only on a $0.3 \mathrm{kPa}$ substrate (Fig. 5a, black line); the latter takes a longer time to differentiate.

\section{Feedback signaling onto the effective substrate adhesion area}

Mechanotransduction pathways may contain positive feedback loops in which integrin engagement activates actomyosin cytoskeleton contractility, resulting in morphological changes affecting the adhesion area of the substrate $[1,17,18,23-27]$. Here we assess the importance of such feedback. Figure 6 shows the relative expression levels of the lineage-specific genes at steady states for a range of substrate stiffness values. In Fig. 6a, we block the feedback from TUBB3 onto the effective substrate adhesion area. We see that the bistability that was observed in Fig. 3 is no longer present: no hysteresis effect can be seen when the substrate stiffness is increased or decreased (illustrated by the blue and red lines). Thus, no mechanical memory effect remains for TUBB3 during MSCs differentiation. Similar results are obtained for PPARG (Fig. 6b), MYOD1 (Fig. 6c) and RUNX2 (Fig. 6d) when the final seeding stiffness is $0.9 \mathrm{kPa}, 10 \mathrm{kPa}$ and $16 \mathrm{kPa}$, respectively. The mechanical memory of the genes disappeares when the feedback loops are removed. Collectively our simulation results illustrate that the feedback loops downstream of the stiffness of substrates are necessary for the mechanical memory. a

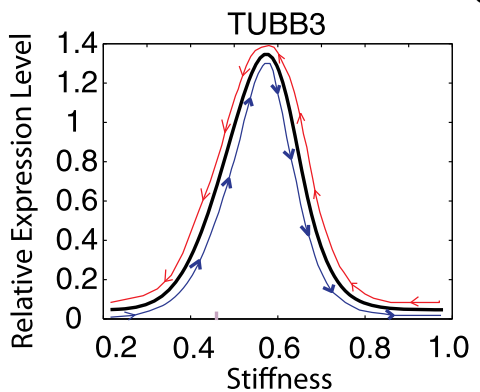

b

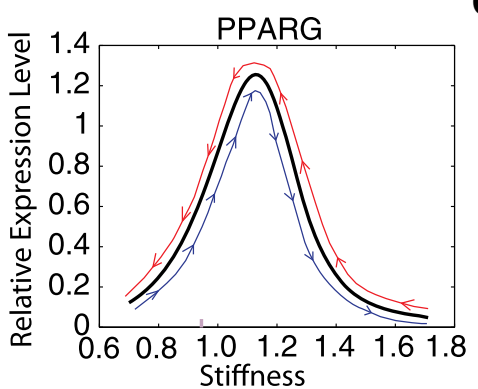

C

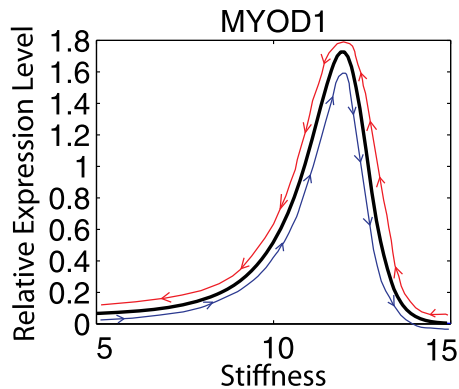

d

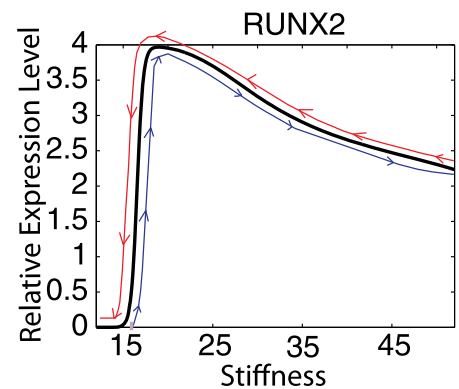

Fig. 6 The MSC network precludes multistability when feedback loops are blocked. Shown are the steady states of TUBB3 (a), PPARG (b), MYOD1 (c), and RUNX2 (d) under different stiffness values. In each figure the x-axis denotes the stiffness and the $y$-axis denotes the relative expression levels of specific lineage genes at steady states (black lines). The blue lines illustrate how the relative gene expression at the steady state changes as the stiffness increases. The red lines illustrate how the relative gene expression level at the steady state changes as the stiffness decreases 


\section{Noise can induce fate switching during MSC differentiation}

There is inherent noise in gene expression dynamics $[43,44]$. We employ a stochastic differential equation (SDE) model (described in Methods) to study the effects of gene expression noise on MSC differentiation [45, 46]. We find that SDE simulations broadly recapitulate the results obtained in the deterministic case, however under certain conditions fate switching is observed. In Fig. 7 we simulate a system of SDEs based on the deterministic model with multiplicative noise added to the expression level of each gene; blue and dark green lines describe the relative gene expression under the deterministic model, while pink and black lines describe analogous results under the SDE model. We vary the initial seeding stiffness while keeping the second seeding stiffness constant at 12 $\mathrm{kPa}$. In the deterministic case, we see that MYOD1 is expressed when the value of the initial stiffness is $12 \mathrm{kPa}$, and not when the value is $34 \mathrm{kPa}$. Conversely, RUNX2 is not expressed at an initial stiffness of $12 \mathrm{kPa}$, but is expressed when the initial stiffness is $34 \mathrm{kPa}$ : here stem cells are differentiating according to mechanical memory.

In the stochastic case, a different picture emerges. First we note that the memory effect observed for osteogenic differentiation in the deterministic case (driven by RUNX2 expression) is preserved under the stochastic model (Fig. 7 black line). However, in the stochastic case, at $12 \mathrm{kPa}, M Y O D 1$ is expressed transiently: as its expression declines to zero, RUNX2 is turned on. Thus noise has induced a fate transition between myogenic and osteogenic lineages. At $34 \mathrm{kPa}$ no such transitions are observed: RUNX2 is expressed constitutively.

\section{Discussion}

Mesenchymal stem cell fate can be controlled by mechanical dosing [1]. Mechanical memory (past mechanical dosing) also affects stem cell fate, particularly when the initial substrate is stiff [8], it is difficult however to experimentally test the effects of mechanical memory over a wide range of culture conditions. Here we have presented a mathematical model that allows such tests to be performed, producing several striking predictions. We first assessed whether the model is able to recapitulate experimental studies, and find that it does agree with evidence showing MSC differentiation into neurons or adipocytes on softer substrates, and myocytes or osteoblasts on stiffer substrates. We then analyzed model behavior over longer timescales, and found that a mechanical memory region exists for each of these MSC-derived cell lineages, with substantial variation in the memory stiffness range for each cell fate. Previously, a memory region has only been observed during osteogenic differentiation, and even then, only qualitative assessment of its behavior was made. We are able to provide bounds on the substrate stiffness ranges permissive of memory effects for all four lineages.

Upon re-seeding MSCs onto a second substrate, the stem cells differentiate according to mechanical memory under certain conditions. We predict that (in addition to the stiffness of the first substrate) the duration of the first seeding also directly influences stem cell memory. By changing only the duration of the initial seeding we can directly influence cell fate. The number of fates accessible to the MSC can also be controlled by the final seeding stiffness. Landscape analysis demonstrates that,

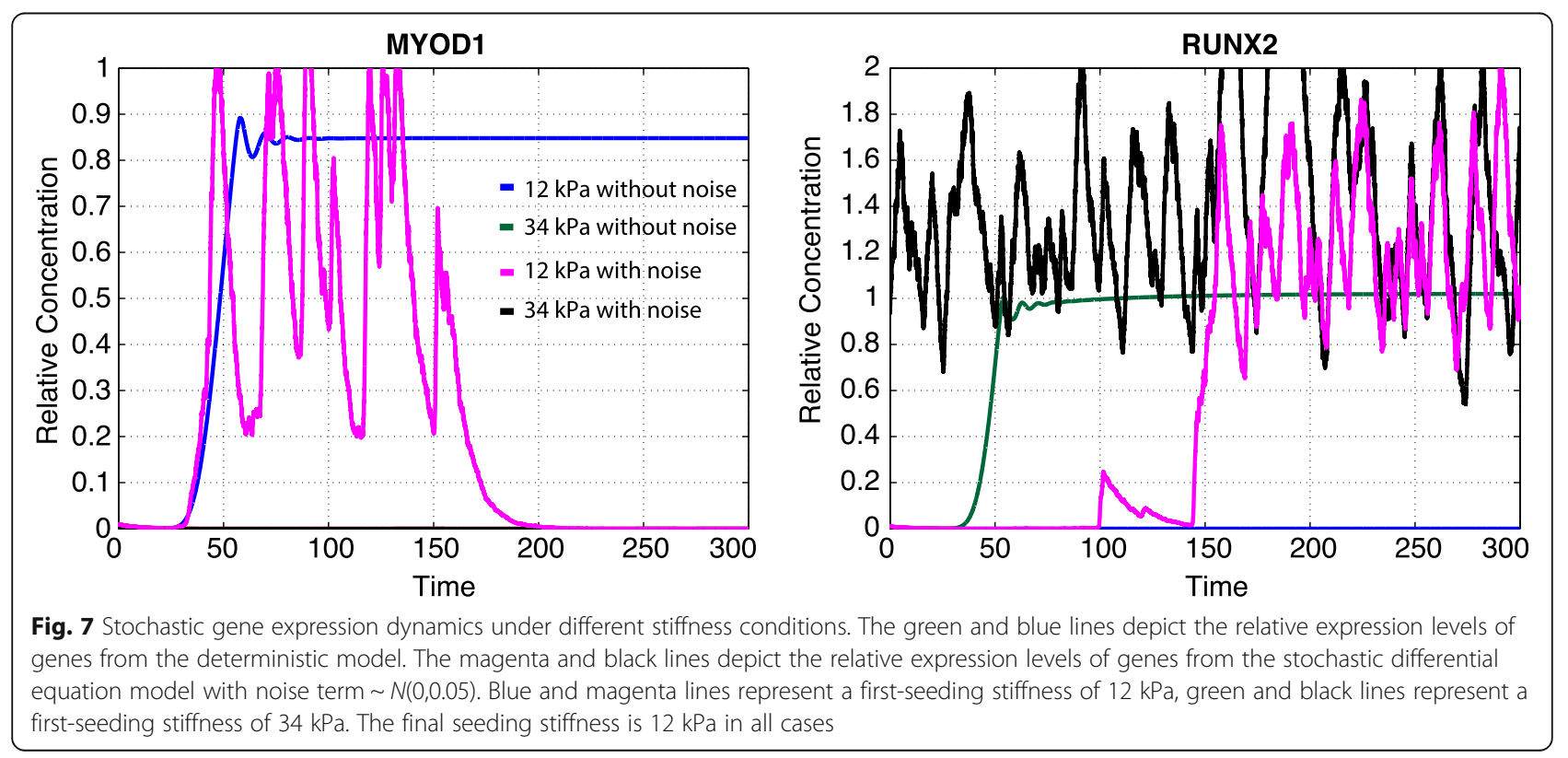


for a constant first seeding stiffness and duration, a higher second seeding stiffness limits the number of MSC fates accessible, and that a sufficiently low final seeding stiffness is permissive of differentiation into all four cell fates. We also found that the feedback loop connecting lineage-specific genes to the effective surface adhesion area is critical for the mechanical memory of MSC differentiation. This might be due to integri$\mathrm{n}$-substrate binding, or morphological changes that occur upon differentiation $[1,3,7,17]$.

As well as their direct relevance for in vitro studies, our model predictions also have important implications for the design of regenerative therapeutics. A major challenge here is lack of precision in cell fate control following transplantation. A better understanding of the relationship between mechanical conditions, culture duration, and stem cell fates is needed. By defining the substrate stiffness limits that regulate MSC fates, this study provides means to design experimental protocols that constrain cells to be confined within fate boundaries, thus avoiding differentiation towards an undesirable fate [47-50]. Mechanical memory could be employed advantageously here, e.g. by preconditioning MSCs via mechanical dosing. An improved understanding of the MSC mechanotransduction pathway will also affect our ability to control multipotency, and should enable us to better culture undifferentiated MSCs in vitro.

In order to study additional effects of the mechanotransduction pathway on stem cell fate, a model that describes a larger regulatory network is needed. Cell-cell interactions have not yet been incorporated into our model, although there is a large body of work detailing the importance of the microenvironment (i.e. the effects of cell-cell interactions and of the niche) on stem cell differentiation [30, 51]. In addition, we have chosen a small set of four lineagespecific genes in order to minimize the size of the model's parameter space. Clearly a greater number of genes are involved in the regulation of MSC fate; without a description of this larger transcriptional network we will not be able to describe nuances of mechanically-induced MSC fate dynamics. However, we believe that the dynamics - and the attractors corresponding to differentiated cell states observed here constitute core pathway mechanisms that would still underlie cell fate decisions in a larger network.

\section{Conclusions}

In this study we sought to investigate the mechanisms of control exerted via mechanical forces upon mesenchymal stem cells during culture and differentiation. Simulations of the gene expression dynamics under different mechanical dosing conditions have led to several predictions. We found that non-cooperative gene regulation is the most plausible mechanism to describe MSC differentiation and we predict that mechanical memory is a general mechanism affecting all of the MSC-derived lineages in this model. We found that the duration of the initial culture and the substrate stiffness during this initial culture are particularly crucial in determining the MSC fates. In addition, we were able to show that a lower final-seeding substrate stiffness permitted a greater number of MSC fates.

Through careful analysis, the ever-expanding body of high-throughput transcriptomic data will enable the study of ever-more complex gene networks. Both the MSC fate transcriptional network structure and the dynamics of the network need to be inferred from data. Spatial interactions, e.g. arising from niche-mediated effects on MSCs, may necessitate a move towards a suitable model framework such as partial differential equations or cell-based (e.g. Cellular Potts) models. Once a clearer picture emerges, it will be possible to extend our model with the incorporation of relevant new signaling interactions. In doing so, we hope to provide further insight into the complex networks of regulation underpinning mesenchymal stem cell fate.

\section{Methods}

\section{A dynamical model of mesenchymal stem cell fate}

We model a simplified gene regulatory network that underpins MSC fate with ordinary differential equations (ODEs) [31, 32].

$$
\begin{aligned}
\frac{d[S A A]}{d t}= & \underbrace{k_{1} \frac{\left(S / K_{1}\right)^{n_{1}}+\left([\text { TUBB3 }] / K_{2}\right)^{n_{2}}}{1+\left(S / K_{1}\right)^{n_{1}}+\left([T U B B 3] / K_{2}\right)^{n_{2}}}}_{[10} \\
& +\underbrace{k_{2} \frac{\left(S / K_{3}\right)^{n_{3}}+\left([P P A R G] / K_{4}\right)^{n_{4}}}{1+\left(S / K_{3}\right)^{n_{3}}+\left([P P A R G] / K_{4}\right)^{n_{4}}}}_{[11} \\
& +\underbrace{k_{3} \frac{\left(S / K_{7}\right)^{n_{7}}+\left([R U N X 2] / K_{8}\right)^{n_{8}}}{1+\left(S / K_{7}\right)^{n_{7}}+\left([R U N X 2] / K_{8}\right)^{n_{8}}}-d_{1}}_{k_{4} \frac{\left(S / K_{5}\right)^{n_{5}}+\left([M Y O D 1] / K_{6}\right)^{n_{6}}}{1+\left(S / K_{5}\right)^{n_{5}}+\left([M Y O D 1] / K_{6}\right)^{n_{6}}}}[S A A]
\end{aligned}
$$

$$
\begin{aligned}
& \frac{d[Y A P T A Z]}{d t}=\underbrace{k_{5}[S A A]}_{1}-d_{2}[Y A P T A Z] \\
& \frac{d[\text { TUBB }]}{d t}=\underbrace{k_{6} \frac{\left([S A A] / K_{9}\right)^{n_{9}}}{1+\left([S A A] / K_{9}\right)^{n_{9}}+\left([Y A P T A Z] / K_{10}\right)^{n_{10}}}}_{2,3}-d_{3}[\text { TUBB3] }
\end{aligned}
$$




$$
\frac{d[\text { PPARG }]}{d t}=\underbrace{k_{7} \frac{\left([S A A] / K_{11}\right)^{n_{11}}}{1+\left([S A A] / K_{11}\right)^{n_{11}}+\left([Y A P T A Z] / K_{12}\right)^{n_{12}}}}_{4,5}-d_{4}[\text { PPARG }]
$$

$$
\frac{d[M Y O D 1]}{d t}=\underbrace{k_{8} \frac{\left([Y A P T A Z] / K_{13}\right)^{n_{13}}}{1+\left([S A A] / K_{14}\right)^{n_{14}}+\left([Y A P T A Z] / K_{13}\right)^{n_{13}}}}_{6,7}-d_{5}[\text { MYOD1 }]
$$

$$
\frac{d[\text { RUNX2 }]}{d t}=\underbrace{k_{9} \frac{\left([\text { YAPTAZ }] / K_{15}\right)^{n_{15}}}{1+\left([S A A] / K_{16}\right)^{n_{16}}+\left([\text { YAPTAZ }] / K_{15}\right)^{n_{15}}}}_{[8,9}-d_{6}[\text { RUNX2 }]
$$

Where $S$ and [SAA], are the relative levels of the stiffness (input to the system) and of the effective stiffness adhesion area, respectively. [YAPTAZ], [TUBB3], [PPARG], [MYOD1], and [RUNX2] denote the relative concentrations of YAP/TAZ, TUBB3, PPARG, MYOD1, and RUNX2. Since concentration and time in the model are given in relative units, i.e. are dimensionless, then all parameters in the above equations are also dimensionless. $d_{i}(i=1,2, \ldots, 6)$ in Eqs. $(1,2,3,4,5$ and 6$)$ are the degradation rates of the corresponding genes/factors. The terms denoted by the label $(1,2, \ldots, 9)$ under the brackets in Eqs. (1,2,3,4,5 and 6) are the active/inhibitive regulations acting on [SAA], [YAPTAZ], [TUBB3], [PPARG], $[M Y O D 1]$, and [RUNX2], where the numbers in rectangle boxes are consistent with the circled indices shown in Fig. 2 [52]. All values of parameters in Eqs. $(1,2,3,4,5$ and 6) shown in Table 2 are estimated or approximated according to the behaviours that we sought to describe. Parameters values are fit to qualitative features of the biological system $[1,6,8,9,19]$ (See Additional file 1). The data required performing full inference of the parameters are as-yet unavailable, however the results of our sensitivity analysis show that the models results do not depend crucially on specific values of parameters of the model.

\section{Cooperative regulation model}

The terms $(10,11,12,13)$ in Eq. (1) are based on the noncooperative regulations of MSCs stiffness sensing. Meanwhile, we model the regulations as the cooperative one and Eq. (1) is rewritten below [53].

Rehfeldt et al showed the "switch-like" nonlinear relationship between $\mathrm{S}$ and SAA expanding from $0.5 \mathrm{kPa}$ to much large stiffness (>60 kPa) and TUBB3, PPARG, $M Y O D 1$, and RUNX2 are turned on in their specific ranges of stiffness, which are relatively disjoint $[52,53]$. In particular, the stiffness range for the myogenic differentiation is far away from the one for adipogenic differentiation. Based the properties of the system, we can rewrite our model into four different submodels under the corresponding stiffness ranges. They are shown as follows.

$$
\frac{d[S A A]}{d t}=k_{1} \frac{\left(S / K_{1}\right)^{n_{1}}+\left([T U B B 3] / K_{2}\right)^{n_{2}}}{1+\left(S / K_{1}\right)^{n_{1}}+\left([T U B B 3] / K_{2}\right)^{n_{2}}}-d_{1}[S A A]
$$

$$
\frac{d[S A A]}{d t}=k_{1} \frac{\left(S / K_{1}\right)^{n_{1}}+\left([P P A R G] / K_{3}\right)^{n_{3}}}{1+\left(S / K_{1}\right)^{n_{1}}+\left([P P A R G] / K_{3}\right)^{n_{3}}}-d_{1}[S A A]
$$

$$
\frac{d[S A A]}{d t}=k_{1} \frac{\left(S / K_{1}\right)^{n_{1}}+\left([M Y O D 1] / K_{4}\right)^{n_{4}}}{1+\left(S / K_{1}\right)^{n_{1}}+\left([M Y O D 1] / K_{4}\right)^{n_{4}}}-d_{1}[S A A]
$$

$$
\frac{d[S A A]}{d t}=k_{1} \frac{\left(S / K_{1}\right)^{n_{1}}+\left([R U N X 2] / K_{5}\right)^{n_{5}}}{1+\left(S / K_{1}\right)^{n_{1}}+\left([R U N X 2] / K_{5}\right)^{n_{5}}}-d_{1}[S A A]
$$

The difficulty is to determine the values of $K_{1}$. If $K_{1}$ is less than 1000, the hill function in Equation (7) is saturated for high stiffness levels $(>10,000)$ and it means that the models cannot distinguish the myogenic differentiation and osteogenic differentiation since Eqs. (10 and 11) both approach the limit $\frac{d[S A A]}{d t}=k_{1}-d_{1}[S A A]$. If $K_{1}$ is greater than 10,000 , then the model cannot describe the system for low stiffness levels $(<1000)$ with that TUBB3 and PPARG cannot express under the low stiffness levels since Eqs. (8 and 9) will respectively approach the limit:

$$
\begin{aligned}
& \frac{d[S A A]}{d t}=k_{1} \frac{\left([\text { TUBB3 }] / K_{2}\right)^{n_{2}}}{1+\left([\text { TUBB3 }] / K_{2}\right)^{n_{2}}}-d_{1}[S A A] \\
& \frac{d[S A A]}{d t}=k_{1} \frac{\left([P P A R G] / K_{3}\right)^{n_{3}}}{1+\left([P P A R G] / K_{3}\right)^{n_{3}}}-d_{1}[S A A]
\end{aligned}
$$

Thus the cooperative regulation model is unable to accurately describe the MSC differentiation system over the range of stiffness values considered.

$$
\frac{d[S A A]}{d t}=\underbrace{k_{1} \frac{\left(S / K_{1}\right)^{n_{1}}+\left([T U B B 3] / K_{2}\right)^{n_{2}}+\left([P P A R G] / K_{3}\right)^{n_{3}}+\left([M Y O D 1] / K_{4}\right)^{n_{4}}+\left([R U N X 2] / K_{5}\right)^{n_{5}}}{1+\left(S / K_{1}\right)^{n_{1}}+\left([T U B B 3] / K_{2}\right)^{n_{2}}+\left([P P A R G] / K_{3}\right)^{n_{3}}+\left([M Y O D 1] / K_{4}\right)^{n_{4}}+\left([R U N X 2] / K_{5}\right)^{n_{5}}}-d_{1}[S A A]}_{10,11,12,13}
$$




\section{Sensitivity analysis}

In order to calculate the sensitivities of the parameters shown in Table 2 with respect to the memory and multistability of the system, we sample 1000 values between $0.2 \mathrm{kPa}$ and $42 \mathrm{kPa}$; they are taken as the stiffness of the system and they are vectorized as the stiffness vector $S_{b}$. We then calculate the steady states, $Q_{b}^{\text {Upper }}$ and $Q_{b}^{\text {Lower }}$, corresponding to the steady states on the lower bifurcation branch (indicated by blue arrowhead lines in Fig. 3c-f, and to the steady states on the upper bifurcation branch (indicated by red arrowhead lines in Fig. 3c-f) for each of the genes: TUBB3, PPARG, MYOD1, and RUNX2, using the parameters in Table 2. In order to calculate the sensitivity of each parameter, we perturbe it 1000 times under the constraint of $\mathrm{CV}$ (coefficient of variance) $=0.05$, and calculate the steady states $Q_{P}^{\text {Upper }}$ (with the same initial conditions as $Q_{b}^{\text {Upper }}$ ), and $Q_{p}^{\text {Lower }}$ (with the same initial conditions as $Q_{b}^{\text {Lower }}$ ). We perform such comparisons - for each of the four genes - for a total of 41 parameters and 1000 perturbations, thus for the parameter vector $P_{i}^{j}(i=1,2, \ldots$, $41 ; j=1,2, \ldots, 1000)$, i.e. the $j$-th perturbation of the $i$ th parameter. We count the number $\left(N_{i}\right)$ of $P_{i}^{j}$ that satisfies $\quad\left\|Q_{P}^{\text {Upper }}-Q_{b}^{\text {Upper }}\right\|_{2}+\left\|Q_{P}^{\text {Lower }}-Q_{b}^{\text {Lower }}\right\|_{2}<$ TOL.The tolerance, TOL, is set such the perturbed parameter vector gave rise to the same number of steady states as for the unperturbed case (i.e. multistability and the memory effect is maintained; we set $T O L=4)$. The robustness $R_{i}$ of the $i$-th parameter is defined as $\frac{N_{i}}{10} \%$ and the sensitivity $S_{i}$ of the i-th parameter is $1-\frac{N_{i}}{10} \%$. The robustness values for each of the 41 parameters are shown in the bar graph (See Fig. $3 \mathrm{~g}$ ) and the index of the parameters in the graph is consistent with the one in Table 2. Four of them are sensitive than the rest and they are marked by yellow arrows in the following bar graph.

\section{Steady state analysis}

We compute the steady states of the dynamical system under different $S$ in Eqs. (1, 2, 3, 4, 5 and 6). Here we use the continuation method to compute the steady states and their branches $[54,55]$.

Landscape potential using a mean field self-consistent approximation and Gaussian approximation

Here we derive an approximation for the potential energy of the system. Starting from the Fokker-Planck equation, we calculate the steady state probability distributions using a self-consistent mean field method [56-58]. The probability function $P(X, t)$ satisfies the following diffusion equation:

$$
\begin{aligned}
\frac{\partial P(X, t)}{\partial t}= & -\frac{\partial}{\partial X}[F(X, S) P(X, t)] \\
& +D \frac{\partial^{2}}{\partial X^{2}}[d(X) P(X, t)]
\end{aligned}
$$

where $F(X, S)$ and $d(X)$ are the drift and diffusion part respectively and the noise is weak, i.e. $D<<1$. Note that $\mathrm{X}$ is a vector of species ([SAA],[YAPTAZ],[TUBB3],[P$P A R G],[M Y O D 1],[R U N X 2])$ but we have dropped the arrow notation for convenience below. We factor the original probability function using the self-consistent mean field approach [59], $P(X, t)=\prod_{i=1}^{n} P\left(X_{i}, t\right)$ to reduce the computational complexity of solving the original equation on the probability, similar to a previous study [57]. We use the Gaussian distribution to approximate the true distribution [57], leading to a description for the mean and variance of the gene expression:

$$
\begin{aligned}
& \bar{X}^{\prime}(t)=F(\bar{X}(t), S) \\
& \sigma^{\prime}(t)=\sigma(t) A^{T}(t)+A(t) \sigma(t)+2 D[\bar{X}(t)]
\end{aligned}
$$

where $\bar{X}$ is the mean value of $X(t), \sigma(t)$ is the variance matrix, the matrix element $\alpha_{i j}(t)$ of $A(t)$ is $\frac{\partial F_{i}(\bar{X}(t))}{\partial \bar{X}_{j}(t)}$, i.e. A is the Jacobian matrix.

Since we consider the steady states, then we need to compute $\bar{X}^{(j)}(\infty)$ and $\sigma^{(j)}(\infty)$ from $\bar{X}^{\prime}(t)=0$ and $\sigma^{\prime}(t)=$ 0 , for $j=1,2, \ldots, m$ respectively, where $m$ is the number of basins of attraction. We consider only diagonal elements of $\sigma^{(j)}(\infty)$ from mean field splitting approximation. For each variable $\bar{X}_{i}^{(j)}(\infty)$, the probability distribution can be estimated using the mean and variance and based on Gaussian approximation [57, 60].

$$
P^{(j)}\left(X_{j}, \infty\right)=\frac{1}{\sqrt{2 \pi \sigma^{(j)}}(\infty)} \exp \left[-\frac{\left[X_{i} \bar{X}_{i}^{(j)}(\infty)\right]^{2}}{2 \sigma^{(j)}(\infty)}\right]
$$

If $m=1$, we can use Eq. (6) to compute the probability distribution of the single basin of attraction. If $m>1$, then the system permits multistability, and for each basin of attraction we compute its probability distribution. The probability function thus becomes a weighted sum of the probabilities given for each basin of attraction,

$$
P\left(X_{i}, \infty\right)=\sum_{j=1}^{m} \omega_{j} P^{(j)}\left(X_{i}, \infty\right)
$$

where $\omega_{j}$ is the weighting coefficient of the $j$-th basin. Assume $m$ attractors, then the number of simulations that end up in each attractor is $N_{1}, N_{2}, \ldots, N_{m}$. The weighting coefficient for the $j$-th basin is then calculated 
as $\omega_{j}=N_{j} / \sum_{i=1}^{m} N_{i}$. Finally, we calculate the potential landscapes based on $U(X)=-\ln P(X, \infty)[61,62]$.

\section{A stochastic differential equation model}

A stochastic differential equation (SDE) model for the regulatory network can be constructed via the addition of a noise term $[43,45,46,63]$ :

$$
d X(t)=F(X(t), S) d t+\eta X(t) d W(t)
$$

where $W(t)$ denotes the scalar white noise (or Wiener process), and $\eta$ is the noise coefficient.

\section{Additional file}

Additional file 1: Supplementary information. This PDF contains supplementary results, references and Figures S1-S3 that are not included in the main text. (PDF $191 \mathrm{~kb}$ )

\section{Abbreviations}

ECM: Extracellular matrix; MSC: Mesenchymal stem cell; MYOD1: Myogenic Differentiation 1; ODE: Ordinary differential equation; PPARG: Peroxisome proliferator-activated receptor gamma; RUNX2: Runt-related transcription factor 2; SAA: Effective stiffness adhesion area; SDE: Stochastic differential equation; TAZ: Transcriptional coactivator with PDZ-binding motif; TCPS: Tissue culture polystyrene; TUBB3: The gene encode Tubulin beta-3 chain; YAP: Yes-associated protein

\section{Acknowledgements}

Not applicable.

\section{Funding}

QN was partially supported by NSF grants DMS1161621, and DMS1562176, and NIH grants P50GM076516, R01GM107264, and R01NS095355. WZ was partially supported by the NIH grant 1DP2CA195763.

\section{Availability of data and materials}

The dataset supporting the conclusions of this article is included within the article and its Additional file 1.

\section{Authors' contributions}

The overall work was conceived and designed by TP, LL, WZ and QN. Simulations and implementations of the model were carried out by TP. Data analysis was performed by TP, LL, ALM, CWW, WZ, and QN. TP and LL wrote the first draft of the manuscript, and TP, LL, ALM, CWW, WZ, and QN together finalized the manuscript. All authors have read and approved the final version of the manuscript.

\section{Competing interests}

The authors declare that they have no competing interests.

\section{Consent for publication}

Not applicable.

Ethics approval and consent to participate

Not applicable.

\section{Publisher's Note}

Springer Nature remains neutral with regard to jurisdictional claims in published maps and institutional affiliations.

\section{Author details}

'Department of Mathematics, Center for Complex Biological Systems, and Center for Mathematical and Computational Biology, University of California,
Irvine, CA 92697, USA. ²Department of Pharmaceutical Sciences, Department of Biomedical Engineering, Department of Biological Chemistry, Sue and Bill Gross Stem Cell Research Center, Chao Family Comprehensive Cancer Center \& Edwards Life sciences Center for Advanced Cardiovascular Technology, University of California, 845 Health Sciences Road, Irvine, CA 92697, USA.

Received: 24 November 2016 Accepted: 26 April 2017

Published online: 16 May 2017

\section{References}

1. Engler AJ, Sen S, Sweeney HL, Discher DE. Matrix elasticity directs stem cell lineage specification. Cell. 2006;126:677-89.

2. Guilak F, Cohen DM, Estes BT, Gimble JM, Liedtke W, Chen CS. Control of stem cell fate by physical interactions with the extracellular matrix. Cell Stem Cell. 2009:5:17-26.

3. Trappmann B, Gautrot JE, Connelly JT, Strange DGT, Li Y, Oyen ML, et al. Extracellular-matrix tethering regulates stem-cell fate. Nat Mater. 2012;11:642-9.

4. Khetan S, Guvendiren M, Legant WR, Cohen DM, Chen CS, Burdick JA. Degradation-mediated cellular traction directs stem cell fate in covalently crosslinked three-dimensional hydrogels. Nat Mater. 2013;12:458-65.

5. Ivanovska IL, Shin J-W, Swift J, Discher DE. Stem cell mechanobiology: diverse lessons from bone marrow. Trends Cell Biol. 2015;25(9):523-32

6. Halder G, Dupont S, Piccolo S. Transduction of mechanical and cytoskeletal cues by YAP and TAZ. Nat Rev Mol Cell Biol. 2012;13:591-600.

7. Wen JH, Vincent LG, Fuhrmann A, Choi YS, Hribar KC, Taylor-Weiner H, et al. Interplay of matrix stiffness and protein tethering in stem cell differentiation. Nat Mater. 2014;13:979-87.

8. Yang C, Tibbitt MW, Basta L, Anseth KS. Mechanical memory and dosing influence stem cell fate. Nat Mater. 2014;13(6):645-52.

9. Rehfeldt F, Brown AEX, Raab M, Cai S, Zajac AL, Zemel A, et al. Hyaluronic acid matrices show matrix stiffness in 2D and 3D dictates cytoskeletal order and myosin-II phosphorylation within stem cells. Integr Biol. 2012:4:422-30.

10. Raghunathan VK, Morgan JT, Dreier B, Reilly CM, Thomasy SM, Wood JA, et al. Role of substratum stiffness in modulating genes associated with extracellular matrix and mechanotransducers YAP and TAZ. Invest Ophthalmol Vis Sci. 2013:54:378-86.

11. Azzolin L, Panciera T, Soligo S, Enzo E, Bicciato S, Dupont S, et al. YAP/TAZ incorporation in the $\beta$-Catenin destruction complex orchestrates the Wnt response. Cell. 2014;158:157-70.

12. Burke DP, Kelly DJ. Substrate stiffness and oxygen as regulators of stem cell differentiation during skeletal tissue regeneration: a mechanobiological model. PLoS ONE. 2012;7(7):e40737.

13. Mousavi SJ, Doweidar MH. Role of mechanical cues in cell differentiation and proliferation: a 3D numerical model. PLOS ONE. 2015;10:e0124529.

14. Sun M, Spill F, Zaman MH. A computational model of YAP/TAZ mechanosensing. Biophys J. 2016;110:2540-50.

15. Kang K-T, Park J-H, Kim H-J, Lee H-M, Lee K-I, Jung H-H, et al. Study on differentiation of mesenchymal stem cells by mechanical stimuli and an algorithm for bone fracture healing. Tissue Eng Regen Med. 2011:8:359-70.

16. Stops AJF, Heraty KB, Browne M, O'Brien FJ, McHugh PE. A prediction of cell differentiation and proliferation within a collagen-glycosaminoglycan scaffold subjected to mechanical strain and perfusive fluid flow. J Biomech. 2010;43:618-26.

17. Sun $Y$, Chen CS, Fu J. Forcing stem cells to behave: a biophysical perspective of the cellular microenvironment. Annu Rev Biophys. 2012; 41:519-42.

18. Dupont S, Morsut L, Aragona M, Enzo E, Giulitti S, Cordenonsi M, et al. Role of YAP/TAZ in mechanotransduction. Nature. 2011:474:179-83.

19. Swift J, Ivanovska IL, Buxboim A, Harada T, Dingal PCDP, Pinter J, et al Nuclear lamin-A scales with tissue stiffness and enhances matrix-directed differentiation. Science. 2013;341:1240104-4

20. Hong JH, Hwang ES, McManus MT, Amsterdam A, Tian Y, Kalmukova R, et al. TAZ, a transcriptional modulator of mesenchymal stem cell differentiation. Science. 2005;309:1074-8.

21. Hong $J H$, Yaffe MB. TAZ - A beta-catenin-like molecule that regulates mesenchymal stem cell differentiation. Cell Cycle. 2006;5:176-9.

22. Harada S, Rodan GA. Control of osteoblast function and regulation of bone mass. Nature. 2003:423:349-55

23. Yourek G, Hussain MA, Mao JJ. Cytoskeletal changes of mesenchymal stem cells during differentiation. ASAIO J. 2007;53:219-28. 
24. Kilian KA, Bugarija B, Lahn BT, Mrksich M. Geometric cues for directing the differentiation of mesenchymal stem cells. Proc Natl Acad Sci. 2010;107:4872-7.

25. Maharam E, Yaport M, Villanueva NL, Akinyibi T, Laudier D, He Z, et al. Rho/ Rock signal transduction pathway is required for MSC tenogenic differentiation. Bone Res. 2015;3:15015.

26. Matsuoka F, Takeuchi I, Agata H, Kagami H, Shiono H, Kiyota Y, et al. Morphology-based prediction of osteogenic differentiation potential of human mesenchymal stem cells. PLoS ONE. 2013;8:e55082.

27. Rogov IA, Volkova IM, Kuleshov KV, Savchenkova IP. in vitro myogenic differentiation of bovine multipotent mesenchymal stem cells taken from bone marrow and adipose tissue. Agricult Biol. 2012;6:66-72.

28. Dingal PCDP, Bradshaw AM, Cho S, Raab M, Buxboim A, Swift J, et al. Fractal heterogeneity in minimal matrix models of scars modulates stiff-niche stem-cell responses via nuclear exit of a mechanorepressor. Nat Mater. 2015;14:951-60.

29. Adler M, Mayo A, Alon U. Logarithmic and power law input-output relations in sensory systems with fold-change detection. PLoS Comput Biol. 2014; 10(8):e1003781.

30. Peng T, Peng H, Choi D, Su J, Chang C-C, Zhou X. Modeling cell-cell interactions in regulating multiple myeloma initiating cell fate. IEEE J Biomed Health Inform. 2013;18:484-91.

31. Smolen P. Modeling transcriptional control in gene networks - methods, recent results, and future directions. Bull Math Biol. 2000;62:247-92.

32. Ben-Tabou de-Leon S, Davidson EH. Modeling the dynamics of transcriptional gene regulatory networks for animal development. Dev Biol. 2009;325:317-28

33. LV H, Li L, Sun M, Zhang Y, Chen L, Rong Y, et al. Mechanism of regulation of stem cell differentiation by matrix stiffness. Stem Cell Res Ther. 2015;6:1.

34. Gesztelyi R, Zsuga J, Kemeny-Beke A, Varga B, Juhasz B, Tosaki A. The Hill equation and the origin of quantitative pharmacology. Arch Hist Exact Sci. 2012;66:427-38

35. Gilbert PM, Havenstrite KL, Magnusson KEG, Sacco A, Leonardi NA, Kraft P, et al. Substrate elasticity regulates skeletal muscle stem cell self-renewal in culture. Science. 2010;329:1078-81.

36. Lu D, Luo C, Zhang C, Li Z, Long M. Differential regulation of morphology and stemness of mouse embryonic stem cells by substrate stiffness and topography. Biomaterials. 2014;35:3945-55.

37. Choi B, Park KS, Kim JH, Ko KW, Kim JS, Han DK, et al. Stiffness of hydrogels regulates cellular reprogramming efficiency through mesenchymal-toepithelial transition and stemness markers. Macromol Biosci. 2016;16:199-206.

38. Wang J, Zhang K, Xu L, Wang E. Quantifying the waddington landscape and biological paths for development and differentiation. Proc Natl Acad Sci. 2011;108:8257-62.

39. Baker WL. A review of models of landscape change. Landscape Ecol. Kluwer Academic Publishers; 1989;2:111-33

40. Wang J, Xu L, Wang E. Potential landscape and flux framework of nonequilibrium networks: robustness, dissipation, and coherence of biochemical oscillations. Proc Natl Acad Sci. 2008;105:12271-6.

41. Wang J, Li C, Wang E. Potential and flux landscapes quantify the stability and robustness of budding yeast cell cycle network. Proc Natl Acad Sci. 2010;107:8195-200.

42. Murphy WL, McDevitt TC, Engler AJ. Materials as stem cell regulators. Nat Mater. 2014;13:547-57.

43. Elowitz MB, Levine AJ, Siggia ED, Swain PS. Stochastic gene expression in a single cell. Science. 2002;297:1183-6.

44. Chalancon G, Ravarani CNJ, Balaji S, Martinez-Arias A, Aravind L, Jothi R, et al. Interplay between gene expression noise and regulatory network architecture. Trends Genet. 2012;28:221-32.

45. Chen M, Wang L, Liu CC, Nie Q. Noise attenuation in the ON and OFF states of biological switches. ACS Synth Biol. 2013;2:587-93.

46. Holmes WR, Nie Q. Interactions and tradeoffs between cell recruitment, proliferation, and differentiation affect CNS regeneration. Biophys J. 2014; 106:1528-36.

47. Cox TR, Erler JT. Remodeling and homeostasis of the extracellular matrix: implications for fibrotic diseases and cancer. Dis Model Mech. 2011:4:165-78.

48. Mendez MG, Janmey PA. Transcription factor regulation by mechanical stress. Int J Biochem Cell Biol. 2012:44:728-32.

49. Paszek MJ, Zahir N, Johnson KR, Lakins JN, Rozenberg Gl, Gefen A, et al. Tensional homeostasis and the malignant phenotype. Cancer Cell. 2005;8:241-54.

50. Levental KR, Yu H, Kass L, Lakins JN, Egeblad M, Erler JT, et al. Matrix crosslinking forces tumor progression by enhancing integrin signaling. Cell. 2009;139:891-906.
51. Gattazzo F, Urciuolo A, Bonaldo P. Extracellular matrix: a dynamic microenvironment for stem cell niche. Biochim Biophys Acta. 1840;2014 2506-19.

52. Prinz $\mathrm{H}$. Hill coefficients, dose-response curves and allosteric mechanisms. J Chem Biol. 2010;3:37-44.

53. Ingalls B. Mathematical modelling in systems biology: an introduction. 2013.

54. Allgower EL, Georg K. Numerical continuation methods: an introduction. 2012

55. Dhooge A, Govaerts W, Kuznetsov YA, Meijer HGE, Sautois B. New features of the software MatCont for bifurcation analysis of dynamical systems. Math Comput Model Dyn Syst. 2008:14:147-75.

56. Li Q, Wennborg A, Aurell E, Dekel E, Zou J-Z, Xu Y, et al. Dynamics inside the cancer cell attractor reveal cell heterogeneity, limits of stability, and escape. Proc Natl Acad Sci. 2016;113:2672-7.

57. van Kampen NG. Stochastic processes in physics and chemistry. 1985.

58. Li C, Wang J. Quantifying cell fate decisions for differentiation and reprogramming of a human stem cell network: landscape and biological paths. PLoS Comput Biol. 2013;9:e1003165.

59. Sasai M, Wolynes PG. Stochastic gene expression as a many-body problem. Proc Natl Acad Sci. 2003:100:2374-9.

60. Hu G. Stochastic forces and nonlinear systems. 1994.

61. Li C, Wang J. Landscape and flux reveal a new global view and physical quantification of mammalian cell cycle. Proc Natl Acad Sci. 2014;111:14130-5.

62. Li C, Wang E, Wang J. Landscape topography determines global stability and robustness of a metabolic network. ACS Synth Biol. 2012;1(6):229-39.

63. Allen E. Modeling with Itô stochastic differential equations. 2007.

64. McBeath R, Pirone DM, Nelson CM, Bhadriraju K, Chen CS. Cell shape, cytoskeletal tension, and RhoA regulate stem cell lineage commitment. Dev Cell. 2004;6:483-95.

65. Alarcón C, Zaromytidou A-I, Xi Q, Gao S, Yu J, Fujisawa S, et al. Nuclear CDKs drive smad transcriptional activation and turnover in BMP and TGF- $\beta$ pathways. Cell. 2009;139:757-69.

66. Jeong $H$, Bae S, An SY, Byun MR, Hwang J-H, Yaffe MB, et al. TAZ as a novel enhancer of MyoD-mediated myogenic differentiation. FASEB J. 2010;24:3310-20.

67. Bernabé BP, Shin S, Rios PD, Broadbelt $\amalg$, Shea LD, Seidlits SK. Dynamic transcription factor activity networks in response to independently altered mechanical and adhesive microenvironmental cues. Integr Biol. 2016;8:844-60.

\section{Submit your next manuscript to BioMed Central and we will help you at every step:}

- We accept pre-submission inquiries

- Our selector tool helps you to find the most relevant journal

- We provide round the clock customer support

- Convenient online submission

- Thorough peer review

- Inclusion in PubMed and all major indexing services

- Maximum visibility for your research

Submit your manuscript at www.biomedcentral.com/submit 